\title{
Rapid Recurrence of Unilateral Endometrioma in a Teenager with a Noncommunicating Rudimentary Horn and Unicornuate Uterus
}

\author{
Alicia Martínez-Varea*, Vicente Payá-Amate, José María Vila-Vives, Gema Higueras-García, \\ Antonio Abad-Carrascosa, José María Rubio-Rubio, Rafael Romaguera-Torregrosa \\ Department of Obstetrics and Gynecology, La Fe University Hospital, University of Valencia, Valencia, Spain \\ Email: ${ }^{*}$ martinez.alicia.v@gmail.com
}

Received 12 February 2014; revised 10 March 2014; accepted 17 March 2014

Copyright @ 2014 by authors and Scientific Research Publishing Inc.

This work is licensed under the Creative Commons Attribution International License (CC BY).

http://creativecommons.org/licenses/by/4.0/

(c) (i) Open Access

\begin{abstract}
A 17-year-old nulligravida woman presented to the emergency department complaining of a six months' history of severe dysmenorrhea. The patient underwent a laparoscopic excision of a right ovarian endometrioma in another hospital one year ago, 24 months after menarche at 14 years of age. Transvaginal ultrasonography, magnetic resonance imaging, hysteroscopy as well as laparoscopy revealed a unicornuate uterus with a noncommunicating right rudimentary horn, a dilated right fallopian tube and a novel $40 \mathrm{~mm}$ right ovarian endometrioma. An excision of the right fallopian tube, the rudimentary horn and the endometrioma was performed. Since endometriosis may be originated by retrograde menstruation, an obstructive müllerian malformation should be ruled out at the diagnosis of endometriosis, particularly in adolescents. It may lead clinicians to provide an initial definitive treatment for avoiding rapid recurrent endometriosis.
\end{abstract}

\section{Keywords}

Endometrioma; Müllerian Malformation

\section{Introduction}

Müllerian ducts are a couple of embryologic structures which undergo fusion and resorption in utero in order to originate the uterus, fallopian tubes, cervix, and cranial two-thirds of the vagina. Müllerian malformations result

Corresponding author.

How to cite this paper: Martínez-Varea, A., et al. (2014) Rapid Recurrence of Unilateral Endometrioma in a Teenager with a Noncommunicating Rudimentary Horn and Unicornuate Uterus. Open Journal of Obstetrics and Gynecology, 4, $208-211$. http://dx.doi.org/10.4236/ojog.2014.44034 
from an interrupted development, incomplete fusion or resorption of müllerian ducts. These anomalies are often associated with primary amenorrhea, infertility, obstetric complications, and endometriosis [1] [2]. Approximately $20 \%$ of all müllerian duct anomalies are constituted by the unicornuate uterus. Most of these patients are asymptomatic until menarche or until they become pregnant [3].

Being diagnosed in 6\% - 10\% of women of reproductive age, endometriosis is a painful disease characterized by the presence of endometrial tissue in ectopic locations. Ovaries, pelvic peritoneum, and rectovaginal septum are usually first affected [4]. Here, we report a rapid recurrence of a unilateral ovarian endometrioma in a teenager due to an obstructive müllerian malformation.

\section{Case Report}

An otherwise healthy 17-year-old nulligravida woman presented to the emergency department with a six months' history of severe dysmenorrhea. The patient underwent a laparoscopic excision of a right ovarian endometrioma in another hospital one year ago, 24 months after menarche at 14 years of age.

Transvaginal ultrasonography suggested a normal left ovary, a complete septate uterus or a unicornuate uterus with an adhered right rudimentary horn, and a novel $40 \mathrm{~mm}$ right ovarian endometrioma (Figure 1(A)). Magnetic resonance imaging (MRI) showed a unicornuate uterus and a rudimentary noncommunicating right horn with functional endometrium (Figure 1(B)). Anomalies in urinary tract were not found. Hysteroscopy proved a unicornuate uterus with a left ostium. Communication with rudimentary horn was not found (Figure 1(C)). Laparoscopy confirmed the suspected anatomy. A unicornuate uterus with an adhered right rudimentary horn, a dilated right fallopian tube and a $40 \mathrm{~mm}$ right ovarian endometrioma were revealed (Figure 1(D)). Vasopressin instillation on the rudimentary horn elegantly delineated its margins. A markedly limited right fallopian tubal patency was evidenced by methylene blue injection (Figure $1(E)$ ). Communication of the unicornuate uterus with the rudimentary horn was not found during subsequent hysteroscopy (Figure 1(F)). An excision of the right fallopian tube, the rudimentary horn and the endometrioma were performed (Figure 1(G)). Restoration of the uterine integrity involved as the suture of the uterine scar as the uterine union with right round and broad ligaments (Figure $1(\mathrm{H})$ ).

The patient underwent an uncomplicated postoperative period and was discharged from the hospital two days after surgery for further ambulatory following.

\section{Discussion}

Theories regarding endometriosis pathogenesis include those proposing a nonuterine origin of the disease, such as transformation of peritoneal tissue to ectopic endometrial tissue or development of residual cells from embryologic Müllerian duct migration into endometriotic lesions [4]. A nonendometrial origin for endometriosis is based on the suggested transformation of extrauterine stem cells from bone marrow into endometriotic tissue [4]. Finally, uterine endometrium could originate endometriotic implants by lymphatic and/or haematogenous spread or by reflux [4].

Animal and human evidence strongly supports the theory of retrograde menstruation, originally postulated by Sampson in the 1920s [4]. Both the anatomic distribution of endometriotic lesions, and the high prevalence of endometriosis among adolescent girls with congenital outflow obstruction, favours the retrograde menstruation theory [4]. In this sense, unicornuate uterus has been found in 1/1000 women [2] [5], which may have added a rudimentary horn with or without a functional cavity [1]. This müllerian malformation is associated with endometriosis in up to $40 \%$ of cases [2]. Interestingly, the rudimentary horn is noncommunicating in $75 \%$ of cases [1] [5].

The unicornuate uterus with a rudimentary horn found in our patient results from a defective development and fusion of müllerian ducts [2]. As it is usually accompanied by ipsilateral renal agenesis or ipsilateral pelvic kidney [1], they had to be dismissed by MRI. Infertility and poor obstetric outcomes, such as intrauterine growth restriction, fetal anomalous presentation or preterm delivery, are associated with this müllerian malformation [6]. Obstetric complications in the rudimentary communicating horn, including ectopic pregnancy, miscarriage or uterine rupture, and drawbacks associated with a rudimentary noncommunicating horn with functional endometrium, such as endometriosis, are well known [1]-[3] [5] [6]. Thus, management involves removal of the rudimentary horn with a functional endometrial cavity [1] [6].

The finding of a unilateral recurrent endometrioma in a teenager within her first three postmenarchal years 


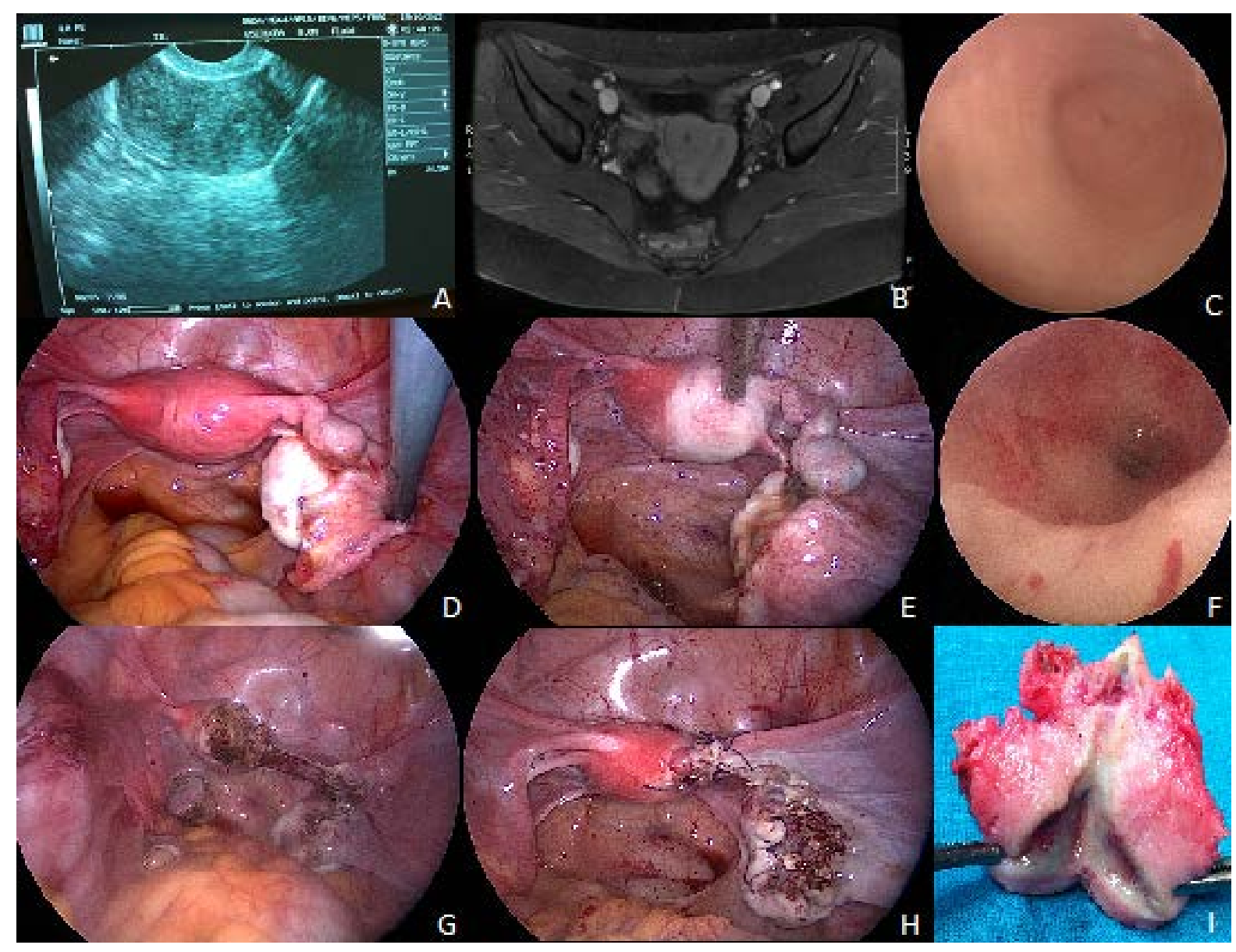

Figure 1. (A) A complete septate uterus or a unicornuate uterus with an adhered right rudimentary horn suggested by transvaginal ultrasonography; (B) A unicornuate uterus and a rudimentary noncommunicating right horn with functional endometrium showed by magnetic resonance imaging; (C) A unicornuate uterus with a left ostium by hysteroscopy; (D) Laparoscopy revealed a unicornuate uterus with an adhered right rudimentary horn, a dilated right fallopian tube and a $40 \mathrm{~mm}$ right ovarian endometrioma; (E) Vasopressin instillation on the rudimentary horn delineated its margins. A markedly limited right fallopian tubal patency evidenced by methylene blue injection; (F) Communication of the unicornuate uterus with the rudimentary horn was not found during subsequent hysteroscopy; (G) Laparoscopic excision of the right fallopian tube, the rudimentary horn and right endometrioma; $(\mathrm{H})$ Suture of the uterine scar and uterine union with right round and broad ligaments restored the uterine integrity; (I) The noncommunicating rudimentary horn with functional endometrial cavity excised.

obliged us to look for an origin of the rapid recurrence of the debilitating disease. Laparoscopy enabled us both to confirm the suspected noncommunicating rudimentary horn with a unicornuate uterus, and to remove the endometrioma and the rudimentary horn. The diagnosis of a noncommunicating rudimentary horn and unicornuate uterus is in concordance with the wide scientific evidence which supports that eutopic endometrium is displaced through fallopian tubes into the peritoneal cavity during menstruation [4]. Thus, after the etiologic treatment, subsequent annual gynaecological follow-up was proposed.

It is well known that estrogens are essential for the development of endometriosis. Thus, the most widely used treatments of endometriosis are sex steroid expression modulators, mainly oral contraceptive pills and GnRH agonists. Anti-inflammatory agents have been classically used to manage the endometriosis-related pain. Nevertheless, they are not associated with a regression of the disease [7]. Actually, patients with obstructive müllerian malformations and endometriosis would take advantage of the preoperative treatment with oral contraceptives to control pain and treat endometriosis if further diagnostic investigations are needed [8].

An obstructive müllerian malformation should be ruled out at the diagnosis of endometriosis, particularly in adolescents, in order to provide an initial definitive treatment for avoid rapid recurrent endometriosis as well as other subsequent complications. 


\section{Declaration of Interest}

The authors report no conflicts of interest.

V. P. A., A. A. C., J. M. R. R., and R. R. T. have contributed to the surgical procedure; A. M. V., J. M. V. V., and G. H. G. have contributed to the design of the case report, together with A. P.; the manuscript has been written by all authors.

All the authors are in agreement with the content of the manuscript. There is no commercial association that might pose a conflict of interest for none of the authors.

There was no involvement of a pharmaceutical/other company.

There were no sponsors in article preparation.

There was no medical writing/editorial assistance with the preparation of the article.

\section{References}

[1] Medeiros. L.R., Rosa, D.D., Silva, F.R., Silva, B.R. and Rosa, M.I. (2011) Laparoscopic Approach of a Unicornuate Uterus with Noncommunicating Rudimentary Horns. ISRN Obstetrics and Gynecology, 2011, 906138. http://dx.doi.org/10.5402/2011/906138

[2] Agarwal, M., Das, A. and Singh, A.S. (2011) Dysmenorrhea Due to a Rare Mullerian Anomaly. Nigerian Journal of Clinical Practice, 14, 377-379. http://dx.doi.org/10.4103/1119-3077.86788

[3] Khati, N.J, Frazier, A. A. and Brindle, K.A. (2012) The Unicornuate Uterus and Its Variants. Journal of Ultrasound in Medicine, 31, 319-331.

[4] Burney, R.O. and Giudice, L.C. (2012) Pathogenesis and Pathophysiology of Endometriosis. Fertility and Sterility, 98, 511-519. http://dx.doi.org/10.1016/j.fertnstert.2012.06.029

[5] Liatsikos, S.A., Tsikouras, P., Souftas, V., Ammari, A., Prassopoulos, P, Maroulis, G., et al. (2010) Diagnosis and Laparoscopic Management of a Rudimentary Uterine Horn in a Teenage Girl, Presenting with Haematometra and Severe Endometriosis: Our Experience and Review of Literature. Minimally invasive Therapy \& Allied Technologies, 19, 241-247. http://dx.doi.org/10.3109/13645701003644491

[6] Atmaca, R., Germen, A.T., Burak, F., Kafkasli, A. (2005) Acute Abdomen in a Case with Noncommunicating Rudimentary Horn and Unicornuate Uterus. JSLS, 9, 235-237.

[7] Soares, S.R., Martínez-Varea, A., Hidalgo-Mora, J.J. and Pellicer, A. (2012) Pharmacologic Therapies in Endometriosis: A Systematic Review. Fertility and Sterility, 98, 529-555. http://dx.doi.org/10.1016/j.fertnstert.2012.07.1120

[8] Elliott, J.E., Abduljabar, H. and Morris, M. (2011) Presurgical Management of Dysmenorrhea and Endometriosis in a Patient with Mayer-Rokitansky-Kuster-Hauser Syndrome. Fertility and Sterility, 96, e86-89. http://dx.doi.org/10.1016/j.fertnstert.2011.06.006 